\section{Análise dos determinantes que influenciam o tempo para o início do tratamento de mulheres com câncer de mama no Brasil}

\author{
Determinants of the time between breast cancer \\ diagnosis and initiation of treatment in \\ Brazilian women
}

\author{
Análisis de los determinantes que influyen en el \\ tiempo para el inicio del tratamiento en mujeres \\ con cáncer de mama en Brasil
}

Giselle Coutinho Medeiros 1

Anke Bergmann 1

Suzana Sales de Aguiar 1

Luiz Claudio Santos Thuler 1
${ }_{1}$ Instituto Nacional de Câncer José Alencar Gomes da Silva, Rio de Janeiro, Brasil.

Correspondência L. C. S. Thuler

Instituto Nacional de Câncer José Alencar Gomes da Silva. Rua André Cavalcanti 37 2 o andar, Rio de Janeiro, $R J$ 20231-050, Brasil.

lthuler@inca.gov.br

\begin{abstract}
This study aimed to analyze the time elapsed between breast cancer diagnosis and initiation of treatment in woman treated from 2000 to 2011 in the Brazilian public health system and to identify factors associated with delayed onset of treatment. This retrospective cohort study included 137,593 women diagnosed in 239 hospitals in Brazil from 2000 to 2011. In $63.1 \%$ of cases the time between diagnosis and treatment was 60 days. Delayed treatment was associated with nonwhite skin color $(O R=1.18$; 95\%CI: 1.13-1.23), single marital status (OR = 1.05; 95\%CI: 1.01-1.09), less than eight years of schooling $(O R=1.13 ; 95 \% C I: 1.08-1.18)$, earlystage disease $(O R=1.27 ; 95 \% C I: 1.22-1.32)$, treatment from 2006 to 2011 (OR = 1.54; 95\%CI: 1.47-1.60), and patients in the public health system $(O R=1.19 ; 95 \%$ CI: 1.13-1.25). Stratified analysis showed variability of factors between regions of Brazil. The identification of factors associated with delayed initiation of breast cancer treatment can support the development of interventions targeted to specific population groups.
\end{abstract}

Breast Neoplasms; Time-to-Treatment; Hospital Records

\section{Resumo}

Este estudo teve como objetivo analisar o intervalo de tempo entre o diagnóstico e o início do tratamento do câncer de mama em mulheres $e$ seus determinantes. Foi realizado um estudo de coorte retrospectiva com 137.593 mulheres diagnosticadas em 239 unidades hospitalares do Brasil entre 2000 a 2011. Em 63,1\% dos casos, o intervalo entre o diagnóstico e o tratamento foi de até 60 dias. No país, as mulheres mais suscetíveis ao atraso foram não brancas $(O R=1,18$; IC95\%: 1,13-1,23), sem companheiro $(O R=1,05$; IC95\%: 1,01-1,09), com menos de oito anos de estudo (OR = 1,13; IC95\%: 1,08-1,18), com doença em estadiamento inicial $(O R=1,27 ;$ IC95\%: 1,22-1,32), tratadas de 2006 a 2011 (OR = 1,54; IC95\%: 1,47-1,60) e provenientes do sistema público de saúde (OR = 1,19; IC95\%: 1,13-1,25). $\mathrm{Na}$ análise estratificada foi observada a variabilidade dos fatores entre as regiões do Brasil. A identificação de fatores associados à demora no início do tratamento poderá possibilitar a elaboração de propostas de intervenções destinadas a grupos populacionais específicos.

Neoplasias de Mama; Tempo para o Tratamento; Registros Hospitalares 


\section{Introdução}

A neoplasia maligna da mama é o segundo tipo de câncer mais incidente no mundo, excetuando-se o câncer de pele não melanoma, e o mais comum entre as mulheres, sendo registrados a cada ano, no mundo, mais de 1,6 milhão de casos novos e pouco mais de 521 mil óbitos pela doença 1. No Brasil, estima-se para o ano de 2015 a ocorrência de 57.120 casos novos de câncer de mama, com um risco estimado de 56,09 casos a cada 100 mil mulheres ${ }^{2}$. Nas últimas três décadas, o câncer de mama tem se constituído na primeira causa de morte por câncer na população feminina, registrando-se aumento das taxas de mortalidade, ajustadas por idade pela população mundial, de 38\% (variação percentual relativa), entre os anos de 1979 a 20113.

Em países desenvolvidos a incidência de câncer de mama mostra-se crescente, acompanhada por declínio nos índices de mortalidade pela doença, estando esse dado associado à utilização de métodos eficientes de detecção precoce e à oferta de tratamento adequado 4 . Entretanto, no Brasil, a mortalidade mostra-se crescente, o que pode ser atribuído ao retardo no diagnóstico e atraso na instituição de terapêutica adequada, o que propicia o crescimento tumoral, tornando o prognóstico da população vulnerável reservado 4,5 .

No âmbito da assistência oncológica a pacientes acometidos por neoplasia mamária, a definição do tempo de atraso pode compreender três momentos distintos: o primeiro ocorre dos primeiros sintomas até a primeira consulta médica; o segundo compreende o período entre a primeira consulta até o primeiro acesso ao serviço de referência especializado; e o terceiro, da primeira avaliação no serviço especializado até o tratamento específico 6,7,8. Recentemente, o Ministério da Saúde do Brasil determinou, por meio de portaria, que o tempo entre o registro do diagnóstico de câncer no prontuário médico e o início do tratamento não deve ultrapassar 60 dias 9.

Existem diferentes critérios para definir o tempo necessário e aceitável para iniciar o tratamento de mulheres acometidas por câncer de mama. Esse fato pode ser atribuído à heterogeneidade do perfil dessas pacientes. Mesmo considerando as que vivem nas mesmas regiões, pode, no entanto, haver realidades sociais, culturais, clínicas e até mesmo de acesso aos serviços distintas 7,10,11. Estudo realizado nos Estados Unidos (Carolina do Norte) com mulheres de baixa renda evidenciou que uma em cada dez mulheres esperaram período maior ou igual a 60 dias para iniciar o tratamento após o diagnóstico, tendo sido esse intervalo associado com diminuição da sobrevida global em $66 \%$ e por câncer de mama em $85 \%$, entre pacientes com estadiamento avançado 12. Estudo que avaliou o impacto da demora da realização de cirurgia com finalidade curativa na sobrevida concluiu que, no câncer de mama, intervalos superiores a 12 semanas estão associados ao aumento da mortalidade pela doença 13. Em contrapartida, análise retrospectiva realizada no Reino Unido obteve resultado diferente dos demais estudos ao sugerir que atrasos superiores a 60 dias não prejudicam significativamente a sobrevivência e que os pacientes que receberam tratamento dentro de um período de 30 dias apresentaram piores resultados do que os que foram tratados em períodos mais tardios 7. Após revisão da literatura científica, observou-se que, no Brasil, existem poucos estudos sobre o atraso para início do tratamento em mulheres com câncer de mama.

Este estudo teve como objetivo analisar o intervalo de tempo entre o diagnóstico e o início do tratamento do câncer de mama em mulheres atendidas entre 2000 e 2011 no sistema público de saúde brasileiro e identificar os fatores associados ao atraso no início do tratamento.

\section{Métodos}

Foi realizado um estudo de coorte retrospectivo de base hospitalar. A população deste estudo foi composta por mulheres com diagnóstico de câncer de mama, cadastradas nos Registros Hospitalares de Câncer (RHC) do Brasil. Os casos registrados nesses bancos de dados são oriundos de 239 unidades hospitalares, sendo $67,7 \%$ localizadas na Região Sudeste, $18 \%$ na Região Nordeste, $12 \%$ na Região Sul, 2,7\% na Região Centro-oeste e 2,6\% na Região Norte.

As variáveis estudadas foram aquelas disponíveis em dois bancos de base administrativa chamados Sistema Integrador (Instituto Nacional de Câncer José Alencar Gomes da Silva. http://wwwl.inca.gov.br/vigilancia, acessado em 28/Mai/2013) e Fundação Oncocentro de São Paulo (FOSP; http://www.fosp.saude.sp.gov. $\mathrm{br} /$ publicacoes/rhc, acessado em 28/Mai/2013).

Foram selecionados casos de mulheres com câncer de mama (CID-10: C50), diagnosticadas entre 1 o de janeiro de 2000 a 31 de dezembro de 2011, sem tratamento oncológico prévio, cujo planejamento terapêutico foi primeiramente estabelecido na unidade cadastrada (casos analíticos seguindo as normas do RHC). Foram excluídas mulheres com idade inferior a 18 anos $(n=90)$ e superior a 80 anos $(n=5.832)$, pelo fato de o câncer de mama ser menos frequente em mulheres nessas faixas etárias. 
Foi estabelecido como desfecho do estudo o intervalo de tempo entre o diagnóstico (data da confirmação anatomopatológica do tumor) e o início do tratamento do câncer de mama, considerando como intervalo ideal o período menor ou igual a 60 dias, e tardio quando superior a 60 dias, utilizando como ponto de corte o prazo estabelecido pelo Ministério da Saúde brasileiro ${ }^{11}$. As datas relativas ao diagnóstico e ao primeiro curso de tratamento estão cadastradas no RHC em dia, mês e ano. O primeiro curso de terapia incluiu todos os tratamentos direcionados para o câncer de mama realizados nos primeiros quatro meses, conforme estabelecido pelo Surveillance, Epidemiology, and End Results Program (SEER) 14 e adotado pelos RHC brasileiros.

Foram analisadas variáveis relativas ao tratamento: modalidades terapêuticas (cirurgia, radioterapia, quimioterapia), data do diagnóstico (em dia/mês/ano), e data do início do tratamento (em dia/mês/ano); variáveis relativas às características sociodemográficas: faixa etária, raça/ cor da pele (de acordo com Instituto Brasileiro de Geografia e Estatística - IBGE), escolaridade (em anos de estudo), consumo de bebida alcoólica (mais de três vezes semanais, independente da quantidade e do total consumido), tabagismo (avaliação do hábito tabágico em qualquer momento da vida), região de residência (em macrorregiões), origem do encaminhamento (Sistema Único de Saúde - SUS e não SUS), estado conjugal (no momento da matrícula); e características clínicas e patológicas: morfologia; tamanho do tumor, status dos linfonodos, presença de metástase, estadiamento clínico (classificação TNM) 15 .

Para a avaliação dos fatores associados ao desfecho, a idade foi dicotomizada em grupos de não idosos (18-59 anos) e idosos (maior ou igual a 60 anos) baseados na definição da Organização Mundial da Saúde (OMS) ${ }^{16}$. As demais variáveis contínuas foram dicotomizadas de acordo com o seu padrão de distribuição. Para a análise descritiva, foi calculada a mediana acompanhada dos valores de mínimo e máximo e para as variáveis categóricas foram calculadas as frequências (absoluta e relativa). A associação entre variáveis independentes e os desfechos foi realizada por análise univariada, por meio de odds ratio (OR) brutas, assumindo-se intervalos de 95\% de confiança (IC95\%). As variáveis relativas ao TNM foram dicotomizadas em estadiamento inicial $(<2 \mathrm{~B})$ e avançado ( $\geq 2 \mathrm{~B}$ ). A variável morfologia não foi utilizada no modelo de associação por não haver plausibilidade biológica que sustente sua relação com o desfecho. A variável consumo de álcool também não foi incluída nessa análise pela impossibilidade de identificar quando o consumo foi elevado ou abusivo. Além disso, o tipo de tratamento recebido não foi considerado no modelo de associação por se tratar do desfecho propriamente dito. As associações com significância clínica ou valor de $\mathrm{p}<0,20$ na análise univariada foram incluídas em modelo de regressão logística pelo método stepwise forward. Os dados foram analisados por meio do pacote estatístico SPSS versão 20.0 (IBM Corp., Armonk, Estados Unidos).

Este estudo foi aprovado pelo Comitê de Ética em Pesquisa do Instituto Nacional de Câncer José Alencar Gomes da Silva (parecer no 128/11), estando de acordo com os princípios éticos estabelecidos pelo Conselho Nacional de Saúde (CNS).

\section{Resultados}

Foram incluídas neste estudo 137.593 mulheres com câncer de mama tratadas entre 2000 e 2011. A mediana da idade foi de 54 anos (18-80 anos), havendo predomínio de mulheres com idade entre 50 e 69 anos (48,1\%), de raça/cor da pele branca $(52,4 \%)$, com Ensino Fundamental incompleto (42,3\%), residentes da Região Sudeste do país $(64,7 \%)$, casadas ou que viviam em união consensual estável (54,3\%). Relataram ser consumidoras de álcool ou ex-consumidoras 15,3\% das mulheres, e tabagistas ou ex-tabagistas $29,7 \%$. Constatou-se que $75,3 \%$ procederam do SUS e que a maioria das mulheres foi diagnosticada entre os anos de 2004 e 2007 (37,2\%), seguidas pelas que receberam diagnóstico entre 2008 e 2011 (37\%) (Tabela 1).

O tipo histológico mais comum foi o carcinoma ductal invasivo $(81,2 \%)$. Predominaram ainda o estádio II (38,9\%), tumores entre 2 e $5 \mathrm{~cm}$ (36,3\%), com linfonodos clinicamente comprometidos $(50,2 \%)$ e sem metástase à distância $(91,7 \%)$. Ao considerarmos o primeiro curso de tratamento realizado nos primeiros quatro meses, a maior parte das mulheres foi submetida à cirurgia $(67,2 \%)$, seguida de quimioterapia (61\%), radioterapia $(39,5 \%)$ e hormonioterapia (23,6\%) (Tabela 2).

O tempo mediano entre o diagnóstico e o início do tratamento foi de 43 dias (Região Sudeste: 44 dias; Nordeste: 43 dias; Sul: 39 dias; Centrooeste: 30 dias; Norte: 49 dias). A diferença entre as regiões foi estatisticamente significante ( $\mathrm{p}<0,001$ ). Em $63,1 \%$ dos casos, o início do tratamento ocorreu em um intervalo de tempo menor ou igual a 60 dias. Entre as pacientes $(n=56.094$ mulheres) que foram encaminhadas ao hospital especializado com diagnóstico de câncer previamente realizado, o tempo mediano entre o diagnóstico e a primeira consulta foi de 35 dias; da primeira consulta até o início do tratamento 
Tabela 1

Características demográficas das mulheres com câncer de mama, cadastradas nos Registros Hospitalares de Câncer. Brasil, 2000-2011 ( $N=137.593)$

\begin{tabular}{|c|c|c|}
\hline Características & $n$ * & $\%$ \\
\hline \multicolumn{3}{|l|}{ Faixa etária } \\
\hline $18-39$ & 15.766 & 11,5 \\
\hline $40-49$ & 36.977 & 26,9 \\
\hline $50-69$ & 66.119 & 48,1 \\
\hline $70-80$ & 18.731 & 13,5 \\
\hline \multicolumn{3}{|l|}{ Raça/Cor da pele } \\
\hline Branca & 38.234 & 52,4 \\
\hline Parda & 28.366 & 38,8 \\
\hline Preta & 5.783 & 7,9 \\
\hline Amarela & 549 & 0,8 \\
\hline Indígena & 81 & 0,1 \\
\hline \multicolumn{3}{|l|}{ Escolaridade } \\
\hline Analfabeto & 8.553 & 9,0 \\
\hline Ensino Fundamental incompleto & 40.166 & 42,3 \\
\hline Ensino Fundamental completo & 18.078 & 19,1 \\
\hline Ensino Médio ou 2o grau & 18.474 & 19,5 \\
\hline Ensino Superior & 9.586 & 10,1 \\
\hline \multicolumn{3}{|l|}{ Consumo de álcool } \\
\hline Sim (consumidor ou ex-consumidor) & 7.565 & 15,3 \\
\hline Não & 41.903 & 84,7 \\
\hline \multicolumn{3}{|l|}{ Tabagismo } \\
\hline Sim (consumidor ou ex-consumidor) & 15.999 & 29,7 \\
\hline Não & 37.791 & 70,3 \\
\hline \multicolumn{3}{|l|}{ Região de residência } \\
\hline Sudeste & 88.864 & 64,7 \\
\hline Nordeste & 24.654 & 18,0 \\
\hline Sul & 16.474 & 12,0 \\
\hline Centro-oeste & 3.728 & 2,7 \\
\hline Norte & 3.558 & 2,6 \\
\hline \multicolumn{3}{|l|}{ Origem do encaminhamento } \\
\hline SUS & 54.062 & 75,3 \\
\hline Não SUS & 13.920 & 19,4 \\
\hline Por conta própria & 3.768 & 5,3 \\
\hline \multicolumn{3}{|l|}{ Estado conjugal } \\
\hline Casado ou união consensual & 39.412 & 54,2 \\
\hline Solteiro & 16.958 & 23,4 \\
\hline Viúvo & 10.955 & 15,1 \\
\hline Separado judicialmente & 5.268 & 7,3 \\
\hline \multicolumn{3}{|l|}{ Ano do diagnóstico } \\
\hline $2000-2003$ & 35.519 & 25,8 \\
\hline $2004-2007$ & 51.190 & 37,2 \\
\hline $2008-2011$ & 50.884 & 37,0 \\
\hline
\end{tabular}

SUS: Sistema Único de Saúde.

* As diferenças encontradas correspondem a perda de informação. 
Tabela 2

Características clínicas das mulheres com câncer de mama. Registros Hospitalares de Câncer. Brasil, 2000-2011 ( $N=137.593)$.

\begin{tabular}{|c|c|c|}
\hline Características & $n$ * & $\%$ \\
\hline \multicolumn{3}{|l|}{ Morfologia } \\
\hline Carcinoma ductal invasivo & 111.719 & 81,2 \\
\hline Outros & 25.874 & 18,8 \\
\hline \multicolumn{3}{|l|}{ Tamanho do tumor } \\
\hline In situ & 4.884 & 4,6 \\
\hline TO (Não há evidência de tumor primário) & 982 & 0,9 \\
\hline $\mathrm{T} 1(<2 \mathrm{~cm})$ & 24.614 & 23,2 \\
\hline $\mathrm{T} 2(2-5 \mathrm{~cm})$ & 38.605 & 36,3 \\
\hline $\mathrm{T} 3(>5 \mathrm{~cm})$ & 15.401 & 14,5 \\
\hline $\begin{array}{l}\text { T4 (invasão da parede torácica/ulceração de pele ou } \\
\text { nódulos cutâneos/carcinoma inflamatório) }\end{array}$ & 21.818 & 20,5 \\
\hline \multicolumn{3}{|l|}{ Linfonodos } \\
\hline NO (ausência de acometimento linfonodal) & 53.465 & 50,2 \\
\hline N1 (linfonodos axilares móveis) & 35.302 & 33,2 \\
\hline N2 (linfonodos axilares fixos/mamários internos) & 14.589 & 13,7 \\
\hline $\begin{array}{l}\text { N3 (linfonodos infra e supraclaviculares/mamários } \\
\text { internos e axilares) }\end{array}$ & 3.102 & 2,9 \\
\hline \multicolumn{3}{|l|}{ Metástase à distância } \\
\hline Sem metástase & 95.933 & 91,7 \\
\hline Com metástase & 8.657 & 8,3 \\
\hline \multicolumn{3}{|l|}{ Estadiamento } \\
\hline 0 & 5.496 & 4,6 \\
\hline । & 20.656 & 17,3 \\
\hline ॥ & 46.489 & 38,9 \\
\hline III & 36.376 & 30,5 \\
\hline IV & 10.356 & 8,7 \\
\hline \multicolumn{3}{|l|}{ Cirurgia isolada ou combinada } \\
\hline Sim & 92.292 & 67,2 \\
\hline Não & 45.040 & 32,8 \\
\hline \multicolumn{3}{|l|}{ Radioterapia isolada ou combinada } \\
\hline Sim & 54.277 & 39,5 \\
\hline Não & 83.055 & 60,5 \\
\hline \multicolumn{3}{|l|}{ Quimioterapia isolada ou combinada } \\
\hline Sim & 83.791 & 61,0 \\
\hline Não & 53.541 & 39,0 \\
\hline \multicolumn{3}{|l|}{ Hormonioterapia isolada ou combinada } \\
\hline Sim & 32.396 & 23,6 \\
\hline Não & 104.936 & 76,4 \\
\hline
\end{tabular}

* As diferenças encontradas correspondem à perda de informação.

decorreram 22 dias. Já para as pacientes ( $\mathrm{n}=$ 81.499 mulheres) que chegaram ao hospital especializado sem diagnóstico de câncer, o tempo mediano entre a primeira consulta e a confirmação diagnóstica foi de 13 dias, e entre o diagnóstico e o início do tratamento decorreram 28 dias.
A análise univariada das variáveis associadas ao atraso entre o diagnóstico de câncer e o início do tratamento, para as macrorregiões e para o país como um todo está apresentada na Tabela 3. Já a análise múltipla estratificada pelas macrorregiões do Brasil pode ser visualizada na Tabela 4. 
Variáveis associadas ao tempo entre o diagnóstico e início do tratamento (por região).

\begin{tabular}{|c|c|c|c|c|c|c|c|c|c|}
\hline \multirow[t]{2}{*}{ Variáveis } & \multicolumn{3}{|c|}{ Região Norte } & \multicolumn{3}{|c|}{ Região Nordeste } & \multicolumn{3}{|c|}{ Região Sudeste } \\
\hline & OR & IC95\% & Valor de $p$ & OR & IC95\% & Valor de $p$ & OR & IC95\% & Valor de $p$ \\
\hline \multicolumn{10}{|l|}{ Idade (anos) } \\
\hline$\geq 60$ vs. $18-59$ * & 0,97 & $0,82-1,14$ & 0,706 & 1,11 & $1,05-1,18$ & $<0,001$ & 1,04 & $1,01-1,07$ & 0,004 \\
\hline \multicolumn{10}{|l|}{ Raça/Cor da pele } \\
\hline Outras ** vs. branca * & 0,96 & $0,77-1,19$ & 0,712 & 1,13 & $1,06-1,21$ & $<0,001$ & 1,23 & $1,18-1,29$ & $<0,001$ \\
\hline \multicolumn{10}{|l|}{ Anos de estudo } \\
\hline$<8$ vs. $\geq 8$ * & 1,10 & $0,95-1,28$ & 0,22 & 1,28 & $1,20-1,37$ & $<0,001$ & 1,25 & $1,20-1,29$ & $<0,001$ \\
\hline \multicolumn{10}{|l|}{ Tabagismo } \\
\hline Sim vs. não * & 1,06 & $0,90-1,26$ & 0,484 & 0,92 & $0,85-1,00$ & 0,073 & 1,10 & $1,05-1,16$ & $<0,001$ \\
\hline \multicolumn{10}{|l|}{ Estado conjugal } \\
\hline Sem vs. com companheiro * & 1,15 & $0,98-1,34$ & 0,089 & 1,07 & $1,01-1,13$ & 0,027 & 1,09 & $1,04-1,15$ & $<0,001$ \\
\hline \multicolumn{10}{|l|}{ Encaminhamento } \\
\hline SUS vs. não SUS * & 0,91 & $0,74-1,10$ & 0,33 & 2,05 & $1,90-2,21$ & $<0,001$ & 1,12 & $1,07-1,18$ & $<0,001$ \\
\hline \multicolumn{10}{|l|}{ Estadiamento clínico } \\
\hline$<\| B$ vs. $\geq \| B$ * & 1,11 & $0,94-1,33$ & 0,194 & 1,23 & $1,16-1,31$ & $<0,001$ & 1,07 & $1,04-1,10$ & $<0,001$ \\
\hline \multicolumn{10}{|l|}{ Ano do diagnóstico } \\
\hline \multirow[t]{3}{*}{ 2006-2011 vs. 2000-2005 * } & 1,47 & $1,28-1,69$ & $<0,001$ & 1,21 & $1,15-1,28$ & $<0,001$ & 1,82 & $1,77-1,87$ & $<0,001$ \\
\hline & \multicolumn{3}{|c|}{ Região Sul } & \multicolumn{3}{|c|}{ Região Centro-oeste } & \multicolumn{3}{|c|}{ Brasil } \\
\hline & OR & $\mathrm{IC} 95 \%$ & Valor de p & OR & IC95\% & Valor de p & OR & IC95\% & Valor de $p$ \\
\hline \multicolumn{10}{|l|}{ Idade (anos) } \\
\hline$\geq 60$ vs. $18-59$ * & 1,09 & $1,02-1,17$ & 0,014 & 1,02 & $0,87-1,20$ & 0,783 & 1,06 & $1,04-1,08$ & $<0,001$ \\
\hline \multicolumn{10}{|l|}{ Raça/Cor da pele } \\
\hline Outras ** vs. branca * & 0,94 & $0,93-1,07$ & 0,363 & 0,72 & $0,60-0,89$ & 0,002 & 1,12 & $1,09-1,16$ & $<0,001$ \\
\hline \multicolumn{10}{|l|}{ Anos de estudo } \\
\hline$<8$ vs. $\geq 8$ * & 1,28 & $1,18-1,39$ & $<0,001$ & 1,20 & $0,99-1,46$ & 0,059 & 1,25 & $1,21-1,28$ & $<0,001$ \\
\hline \multicolumn{10}{|l|}{ Tabagismo } \\
\hline Sim vs. não * & 1,16 & $1,05-1,29$ & 0,005 & 1,11 & $0,85-1,46$ & 0,446 & 1,08 & $1,03-1,12$ & $<0,001$ \\
\hline \multicolumn{10}{|l|}{ Estado conjugal } \\
\hline Sem vs. com companheiro * & 1,02 & $0,94-1,01$ & 0,685 & 1,01 & $0,83-1,23$ & 0,915 & 1,09 & $1,06-1,12$ & $<0,001$ \\
\hline \multicolumn{10}{|l|}{ Encaminhamento } \\
\hline SUS vs. não SUS * & 2,29 & $2,05-2,57$ & $<0,001$ & 0,82 & $0,68-1,00$ & 0,055 & 1,34 & $1,29-1,38$ & $<0,001$ \\
\hline \multicolumn{10}{|l|}{ Estadiamento clínico } \\
\hline$<\| \mathrm{IB}$ vs. $\geq \mathrm{IIB}$ * & 1,60 & $0,99-1,15$ & 0,112 & 1,35 & $1,13-1,61$ & 0,001 & 1,10 & $1,07-1,12$ & $<0,001$ \\
\hline \multicolumn{10}{|l|}{ Ano do diagnóstico } \\
\hline 2006-2011 vs. $2000-2005$ * & 1,07 & $0,99-1,15$ & 0,078 & 1,38 & $1,19-1,60$ & $<0,001$ & 1,57 & $1,53-1,60$ & $<0,001$ \\
\hline
\end{tabular}

IC95\%: intervalo de 95\% de confiança; OR: odds ratio; SUS: Sistema Único de Saúde.

* Categoria de referência;

** Parda, preta, indígena e amarela.

Na última, verificou-se que as mulheres com intervalo de tempo mais longo para o início do tratamento do câncer de mama foram as com mais de 60 anos de idade (regiões Sudeste e Sul), as não brancas (regiões Nordeste e Sudeste), vivendo sem companheiro (regiões Norte e Sudes- te), com menos de oito anos de estudo (regiões Nordeste e Sul), com doença em estadiamento inicial (regiões Nordeste e Sul), que receberam diagnóstico entre os anos de 2006 e 2011 (regiões Norte, Nordeste, Sudeste e Centro-oeste), bem como aquelas encaminhadas pelo SUS (regiões 
Variáveis independentes do modelo de regressão múltipla para o atraso do diagnóstico ao tratamento por macrorregião e no Brasil.

\begin{tabular}{|c|c|c|c|c|c|c|c|c|c|c|c|c|}
\hline \multirow[t]{3}{*}{ Variáveis } & \multicolumn{10}{|c|}{ Macrorregiões do Brasil } & \multicolumn{2}{|c|}{ Brasil } \\
\hline & \multicolumn{2}{|c|}{ Norte } & \multicolumn{2}{|c|}{ Nordeste } & \multicolumn{2}{|c|}{ Sudeste } & \multicolumn{2}{|c|}{ Sul } & \multicolumn{2}{|c|}{ Centro-oeste } & \multirow[b]{2}{*}{$\begin{array}{c}\text { OR *** } \\
\text { (IC95\%) }\end{array}$} & \multirow[b]{2}{*}{$\begin{array}{l}\text { Valor } \\
\text { de } p\end{array}$} \\
\hline & $\begin{array}{c}\text { OR * } \\
\text { (IC95\%) }\end{array}$ & $\begin{array}{l}\text { Valor } \\
\text { de } p\end{array}$ & $\begin{array}{c}\text { OR ** } \\
\text { (IC95\%) }\end{array}$ & $\begin{array}{l}\text { Valor } \\
\text { de p }\end{array}$ & $\begin{array}{c}\text { OR } \\
\text { (IC95\%) }\end{array}$ & $\begin{array}{l}\text { Valor } \\
\text { de } p\end{array}$ & $\begin{array}{c}\text { OR } \\
\text { (IC95\%) }\end{array}$ & $\begin{array}{l}\text { Valor } \\
\text { de } p\end{array}$ & $\begin{array}{c}\text { OR } \\
\text { (IC95\%) }\end{array}$ & $\begin{array}{l}\text { Valor } \\
\text { de p }\end{array}$ & & \\
\hline \multicolumn{13}{|l|}{ Idade (anos) } \\
\hline$\geq 60$ vs. $18-59$ \# & - & - & - & - & $\begin{array}{c}1,06 \\
(1,01-1,12)\end{array}$ & 0,018 & $\begin{array}{c}1,20 \\
(1,06-1,35)\end{array}$ & 0,003 & - & - & - & - \\
\hline \multicolumn{13}{|l|}{ Raça/Cor da pele } \\
\hline Outras \#\# vs. branca \# & - & - & $\begin{array}{c}1,03 \\
(0,93-1,14)\end{array}$ & 0,571 & $\begin{array}{c}1,17 \\
(1,12-1,23)\end{array}$ & $<0,001$ & - & - & - & - & $\begin{array}{c}1,18 \\
(1,13-1,23)\end{array}$ & $<0,001$ \\
\hline \multicolumn{13}{|l|}{ Anos de estudo } \\
\hline$<8$ vs. $\geq 8 \#$ & - & - & $\begin{array}{c}1,19 \\
(1,10-1,29)\end{array}$ & $<0,001$ & - & - & $\begin{array}{c}1,23 \\
(1,10-1,38)\end{array}$ & $<0,001$ & - & - & $\begin{array}{c}1,13 \\
(1,08-1,18)\end{array}$ & $<0,001$ \\
\hline \multicolumn{13}{|l|}{ Tabagismo } \\
\hline Sim vs. não \# & - & - & - & - & - & - & - & - & - & - & - & - \\
\hline \multicolumn{13}{|l|}{ Estado conjugal } \\
\hline Sem vs. com companheiro \# & $\begin{array}{c}1,16 \\
(0,99-1,35)\end{array}$ & 0,074 & - & - & $\begin{array}{c}1,08 \\
(1,03-1,14)\end{array}$ & 0,002 & - & - & - & - & $\begin{array}{c}1,05 \\
(1,01-1,09)\end{array}$ & 0,026 \\
\hline \multicolumn{13}{|l|}{ Encaminhamento } \\
\hline SUS vs. não SUS \# & - & - & $\begin{array}{c}2,06 \\
(1,86-2,28)\end{array}$ & $<0,001$ & $\begin{array}{c}1,07 \\
(1,02-1,13)\end{array}$ & 0,010 & $\begin{array}{c}2,07 \\
(1,70-2,52)\end{array}$ & $<0,001$ & - & - & $\begin{array}{c}1,19 \\
(1,13-1,25)\end{array}$ & $<0,001$ \\
\hline \multicolumn{13}{|l|}{ Estadiamento clínico } \\
\hline$<\| B$ vs. $\geq \| \mathrm{IB} \#$ & - & - & $\begin{array}{c}1,33 \\
(1,23-1,44)\end{array}$ & $<0,001$ & - & - & $\begin{array}{c}1,16 \\
(1,03-1,30)\end{array}$ & 0,011 & - & - & $\begin{array}{c}1,27 \\
(1,22-1,32)\end{array}$ & $<0,001$ \\
\hline \multicolumn{13}{|l|}{ Ano do diagnóstico } \\
\hline 2006-2011 vs. $2000-2005$ \# & $\begin{array}{c}1,21 \\
(1,04-1,41)\end{array}$ & 0,016 & $\begin{array}{c}1,19 \\
(1,10-1,29)\end{array}$ & $<0,001$ & $\begin{array}{c}1,94 \\
(1,85-2,04)\end{array}$ & $<0,001$ & - & - & $\begin{array}{c}1,38 \\
(1,19-1,60)\end{array}$ & $<0,001$ & $\begin{array}{c}1,54 \\
(1,47-1,60)\end{array}$ & $<0,001$ \\
\hline
\end{tabular}

IC95\%: intervalo de 95\% de confiança; OR: odds ratio; SUS: Sistema Único de Saúde.

*Ajustado por estado conjugal;

** Ajustado por raça/cor;

*** Ajustado por região de residência;

\# Categoria de referência;

\# Parda, preta, indígena e amarela.

Nordeste, Sudeste e Sul), As variáveis referentes à idade e ao tabagismo não mostraram significância estatística na análise (Tabela 4). A análise estratificada por macrorregiões do Brasil pode ser visualizada na Tabela 4.

\section{Discussão}

A proposta deste estudo foi analisar o intervalo de tempo entre o diagnóstico e início do tratamento do câncer de mama em mulheres atendidas entre 2000 a 2011 no sistema público de saúde brasileiro e identificar os fatores associados ao atraso no início do tratamento. Em 63,1\% dos casos, o início do tratamento ocorreu em até 60 dias, estando de acordo tanto com as normas do governo brasileiro quanto de outros centros internacionais, que destacam que prazos maiores que 60 a 90 dias para início da terapêutica podem trazer prejuízos às mulheres com câncer de mama 10,12,17,18. Outro estudo realizado no Rio de Janeiro concluiu que os esforços devem ser concentrados na redução dos tempos necessários para agendar a primeira consulta médica e para o esclarecimento do diagnóstico de lesões suspeitas 19. Também em São Paulo, o maior atraso esteve no tempo entre a descoberta de alterações mamárias pela mulher até a procura por atenção médica 20 , indicando que a determinação do Go- 
verno Federal em oferecer tratamento dentro de 60 dias a partir do diagnóstico objetiva corrigir apenas parte desse complexo problema.

Em países como Canadá 21, Polônia 22 e Estados Unidos 12,17, os intervalos de tempo foram inferiores ao descrito neste estudo, sendo de 34,38 , 22 e 25 dias, respectivamente. Entretanto, estudo realizado em Bogotá (Colômbia) encontrou tempo superior, com mediana de 137 dias 23 . Autores norte-americanos que analisaram o intervalo de tempo entre o diagnóstico e o inicio da quimioterapia neoadjuvante concluíram que a média do tempo encontrado foi de 12 semanas 24 . Além disso, o início precoce do tratamento quimioterápico adjuvante é de extrema importância, sobretudo para os subgrupos de pacientes que apresentam estadiamento avançado ao diagnóstico, e para os acometidos por tumores que expressam HER-2. Atrasos maiores que 60 dias para início da terapia cursam com piores resultados de sobrevida 25. Todavia, órgãos como a American Society of Clinical Oncology (ASCO) e a National Comprehensive Cancer Network (NCCN) recomendam que o início da terapia adjuvante deve ocorrer em até 120 dias para pacientes com idade inferior a 70 anos, diagnosticadas em estádios II ou III da doença, e com tumores que não expressam receptores hormonais 26 .

Neste estudo, as características que influenciaram no desfecho para o conjunto do país foram: ano do diagnóstico, estadiamento da doença, origem do encaminhamento, raça/cor da pele, escolaridade e estado conjugal, corroborando, em sua maioria, estudos anteriores $11,17,27,28,29,30$. Porém, na análise múltipla estratificada por macrorregiões do Brasil, os resultados obtidos demonstram diversidade de fatores que interferem no intervalo de tempo entre o diagnóstico e o início do tratamento do câncer de mama. Observou-se que as mulheres residentes das regiões Sul e Sudeste do Brasil que estavam na faixa etária acima dos 60 anos se mostraram mais suscetíveis ao maior intervalo de tempo para início do tratamento. Essa associação não foi encontrada na análise geral do país. Em estudo realizado em Maringá, no Sul do Brasil, foi descrito que as mulheres na faixa etária entre 51 a 60 anos foram as que menos realizaram o exame de mamografia $(p=0,001)$. O atraso em mulheres com idade mais avançada também foi encontrado em estudo realizado no Estado da Louisiana (Estados Unidos), onde para cada dez anos acrescidos na idade, a chance de atraso no tratamento aumentava em $60 \%$ se comparada àquela das mulheres mais jovens 17 . Em mulheres polonesas, constatou-se que o risco de atraso era maior para mulheres acima dos 70 anos de idade (52 dias) quando comparadas com aquelas de 50 a 69 (35 dias) e de 15 a 49 anos (38 dias) 22 . No México, as pacientes acima dos 65 anos demoraram mais para iniciar o tratamento de quimioterapia adjuvante se comparadas às mais jovens 31. Em estudo realizado no Reino Unido, os autores encontraram resultado divergente ao detectar que cerca de $8 \%$ das mulheres com até 50 anos tiveram atrasos maiores que 90 dias se comparadas a mulheres mais velhas, nas quais esse percentual foi de apenas $3 \%{ }^{7}$. Além disso, outro estudo constatou que mulheres com mais de 80 anos iniciavam tratamento mais precocemente que mulheres mais jovens ${ }^{28}$. Na Nova Zelândia, um estudo mostrou que o atraso foi maior para as mulheres em faixa etária menor que 50 anos e maior que 70 anos 32 .

$\mathrm{Na}$ análise múltipla, que considera todo o Brasil, foi observado que mulheres negras, pardas, indígenas e amarelas apresentaram maiores chances de serem tratadas mais tardiamente que as mulheres de raça/cor da pele branca. No entanto, ao considerarmos essa variável por macrorregião, apenas na Região Sudeste encontramos associação da raça/cor da pele com o desfecho estudado. Esse aspecto tem sido estudado por diversos autores que ponderam que o impacto desse fator está consideravelmente atrelado às possibilidades de acesso ao diagnóstico e ao tratamento 18,27,28,29. Publicações brasileiras que analisaram os fatores relacionados às disparidades no acesso à mamografia e ao retardo na confirmação diagnóstica em estados localizados nessas regiões, encontraram resultados distintos com relação à variável 19,33. Em Teresina (Piauí), as mulheres que se autorreferiram como negras e pardas tinham menor chance de ter realizado mamografia se comparadas às brancas $(p=0,03) 33$. Já em estudo realizado no Rio de Janeiro na década passada, embora o atraso no diagnóstico tenha sido mais frequente nas mulheres de cor parda ou negra, esse fator não apresentou significância estatística na análise 19. Em estudo realizado na população norte- americana, os autores encontraram resultado semelhante ao identificarem que $30,1 \%$ de mulheres negras demoraram 30 dias ou mais para iniciar o tratamento, sendo de $64 \%$ (OR = 1,64; IC95\%: 1,40-1,91) o aumento no risco desse atraso. Esse estudo concluiu que em grupos minoritários o atraso é maior do que em mulheres brancas, e que a morosidade na realização do diagnóstico e do tratamento das mulheres negras norte-americanas demonstrou a fragilidade do sistema de saúde dos Estados Unidos em prestar assistência adequada a esse grupo, desde a detecção do câncer até o tratamento paliativo 28 . Autores neozelandeses corroboraram a afirmativa de que há disparidade no acesso 
aos serviços de saúde para grupos minoritários, concluindo que as populações nativas do país compostas por mulheres Maori (37,1 dias) e do Pacífico $(42,8$ dias) foram submetidas à cirurgia primária mais tardiamente que as mulheres de origem europeia (30,4 dias) 32. Resultado semelhante foi compartilhado por outros pesquisadores 18,21,27,29,34. Contudo, em estudo realizado na Louisiana, com 238 mulheres, das quais $82 \%$ eram negras e $18 \%$ brancas, os autores constataram que atraso maior que 60 dias foi mais frequente nas mulheres brancas $(20,9 \%$ versus 11,3\%) 17 .

Na análise geral do Brasil e na Região Sudeste do país, a situação conjugal foi relacionada ao maior intervalo de tempo para o início do tratamento. Nos estudos que avaliaram as etapas do acesso das mulheres com câncer de mama aos serviços de saúde, foi demonstrado que a maior força de associação tem sido encontrada nos intervalos que antecedem o desfecho analisado neste estudo 11,33,35,36. A influência do atraso nas mulheres sem companheiro também foi retratada em estudos que analisaram os fatores relacionados ao retardo no diagnóstico 11. Dentre as hipóteses, está o fato de que mulheres casadas usufruem mais de serviços preventivos de saúde e realizam mais exames, do que as que acumulam responsabilidades no papel de chefe de família e gestora dos recursos financeiros 11. Outra hipótese é que a descrição e discussão dos sinais e sintomas percebidos pela mulher com o companheiro favorece a procura pelo serviço de saúde, aumentando a chance do diagnóstico precoce 35 .

Identificou-se ainda que mulheres das regiões Sul e Nordeste do país, com menos de oito anos de estudo, têm maior probabilidade de acesso tardio ao tratamento do que mulheres com maior grau de instrução. Essa associação também foi percebida na análise geral do Brasil. Em cidade do Nordeste do Brasil foi observado que mulheres analfabetas tinham 6,89 vezes mais chances de não realização do exame de mamografia se comparadas às que concluíram o Ensino Superior 33. Em Maringá, no Sul do Brasil, as mulheres analfabetas tinham menor chance de terem as mamas examinadas clinicamente do que as outras $(0,0005) 37$. Na Dinamarca, pacientes com maior tempo de estudo foram menos suscetíveis a atrasos médicos (OR = 0,23; IC95\%: 0,09-0,63) 30 . Para esses autores, pacientes com maior escolaridade tendem a descrever melhor os sintomas, tornando mais fácil seu percurso pelo sistema de saúde. Outra explicação é a de que a relação entre médicos e pacientes mais instruídos tende a ser melhor, favorecendo uma investigação diagnóstica mais rápida 30 .
Foi possível observar ainda que as mulheres das regiões Nordeste e Sul que tinham doença avançada ao diagnóstico iniciaram o tratamento mais precocemente, assim como na análise do Brasil. Estudo realizado em Joinville (Santa Catarina) demonstra um cenário preocupante das condições de saúde das mulheres no município 38 . Podemos sugerir que diante do panorama atual, o tratamento das mulheres com estadiamento avançado ao diagnóstico seja instituído mais precocemente em razão do agravamento da doença e do impacto negativo no prognóstico e pior sobrevida. No Reino Unido ${ }^{7}$, em $60 \%$ dos casos com comprometimento linfonodal e em $68 \%$ de mulheres com metástase, o tratamento foi instituído em um intervalo de tempo inferior a 30 dias, o que pode ser por causa da procura por atendimento médico mais precoce de pacientes que apresentam doença sintomática e avançada. Uma hipótese é que seja por conta da maior agressividade do câncer em alguns casos. Outra possibilidade é a de que em alguns países europeus os médicos são mais capazes de identificar os casos mais graves ainda no início do processo de diagnóstico e assumir condutas para iniciar o tratamento mais rapidamente 7 . Outros estudos corroboram tal resultado 17,21,22,31. Em estudo que avaliou o impacto do atraso no tratamento de mulheres com tumor triplo negativo, condição que confere pior prognóstico, não se encontrou associação significativa entre o atraso e a diminuição de sobrevida global. Entretanto, observou-se aumento da tendência de pior sobrevida aos pacientes cujos atrasos excederam 90 dias 39 .

Neste estudo, mulheres das regiões Norte, Nordeste, Sudeste e Centro-oeste que iniciaram tratamento entre os anos de 2000 e 2005 tiveram o tratamento instituído mais rapidamente do que as diagnosticadas nos anos posteriores. Essa associação também foi encontrada na análise geral do Brasil. Pode-se supor que, com o aumento da incidência do câncer de mama no país 2,38,40, houve também o aumento da demanda por procedimentos terapêuticos. Essa hipótese pode explicar a maior espera das pacientes diagnosticadas entre 2006-2011 pelo tratamento. Outra sugestão é a de que o progresso das regiões com ampliação da cobertura mamográfica 11 não foi acompanhado por estratégias de estruturação dos serviços de atendimento oncológico. Estudos realizados no Reino Unido 7, Estados Unidos 10 e Canadá 21 também apontaram que ao longo das últimas décadas tem aumentado progressivamente o tempo para início do tratamento do câncer de mama. No Canadá, a espera por cirurgia de câncer de mama aumentou de 29 dias, em 1992, para 42 dias, em 1998, o que pode 
estar relacionado ao aumento da demanda por diagnóstico e tratamento devido ao aumento da incidência do câncer de mama, diminuição dos recursos e mudanças de padrão do atendimento hospitalar 21. No Reino Unido, foi constatado que, de 1976 a 1995, o intervalo de tempo entre a primeira consulta e o tratamento do câncer de mama aumentou de 7 para 13 dias 7 . Aumento no tempo também foi observado por pesquisadores nos Estados Unidos (de 21 dias em 1992 para 32 dias em 2005), o que pode ser em função da otimização da assistência prestada ao longo dos anos com acesso a recursos mais avançados de diagnóstico e tratamento 10. Em outro estudo norte-americano, também foi observado que ocorreu aumento progressivo do intervalo de tempo entre o diagnóstico e início da quimioterapia adjuvante, entre os anos de 2003 a 2009. No entanto, esses autores concluíram que o a aumento do tempo está relacionado à evolução na realização de técnicas diagnósticas e cirúrgicas de maior qualidade devido aos avanços tecnológicos, e criticam as medidas definidas por órgãos de saúde, sugerindo que prazos generalizados poderiam incentivar mudança nas condutas médicas necessárias 24 .

As usuárias do SUS que residiam nas regiões Sul e Nordeste experimentaram maior atraso entre o diagnóstico e inicio do tratamento oncológico, assim como na análise do Brasil. Alguns autores observaram que a distribuição dos serviços é heterogênea em âmbito regional, sobretudo pela distribuição inadequada dos serviços no território brasileiro 11,40,41,42. Com relação ao acesso ao exame mamográfico, entre os anos de 2003 a 2008, foi evidenciado que as mulheres com plano de saúde realizaram mais mamografias do que as sem plano 11. Quando o desfecho estudado foi o diagnóstico tardio, não foi encontrada associação entre o atraso e o tipo de serviço que antecedeu a matrícula em um centro de referência do SUS, embora em 51,9\% dos casos a primeira consulta tenha sido realizada no serviço privado ${ }^{19}$. Tais resultados sugerem lacunas no acesso ao serviço público de saúde. Liedke et al. 43 observaram que os usuários do sistema público de saúde do Brasil têm maior chance de apresentar doença avançada ao diagnóstico do que os que possuem cobertura de serviços privados.

Em todas as macrorregiões do Brasil, o tempo mediano entre o diagnóstico e o início do tratamento foi menor que 60 dias, e variou de 30 dias na Região Centro-oeste a 49 dias na Região Norte. Estudos brasileiros têm demonstrado que na Região Norte, o acesso à mamografia, ao diagnóstico precoce e ao tratamento tem apresentado os piores resultados 11,41,42. Essas barreiras no aces- so aos serviços de saúde podem gerar grandes impactos, já que dados atuais demonstram um aumento anual de $2,4 \%$ nas taxas de mortalidade por essa neoplasia na região 44 .

Por se tratar de um país com grande extensão territorial e distribuição populacional desigual, devemos considerar que esses resultados sofrem influências de questões socioculturais, de acesso e distribuição dos serviços de saúde e do cadastramento de informações assistenciais 11,41,42,45. Alguns autores concluíram que as regiões Norte e Nordeste do país apresentam maior defasagem de cobertura mamográfica e realização de biópsia 11,42.

Também na etapa referente ao tratamento oncológico, a distância percorrida pelas mulheres com câncer de mama e a concentração dos serviços nos grandes centros demonstra a fragilidade da rede assistencial. Oliveira et al. 41 relataram que há indícios de escassez de oferta de tratamento cirúrgico e ambulatorial na maior parte do Brasil, especialmente na Região Norte, e mesmo em regiões onde a oferta dos serviços mostra-se mais elevada, há dificuldades no acesso aos procedimentos terapêuticos.

Este estudo apresenta algumas limitações que podem comprometer a interpretação dos seus resultados. Como a pesquisa foi realizada em fonte de dados de base secundária, possível viés de informação e seleção deve ser considerado. Na base de dados utilizada, não havia informação disponível que permitisse calcular o tempo de atraso em sua totalidade, incluindo o intervalo de tempo entre os primeiros sintomas até a primeira consulta médica, o intervalo entre a primeira consulta até o primeiro acesso ao serviço de referência especializado, e da primeira avaliação no serviço especializado até o tratamento específico. A inclusão de casos de câncer de mama registrados em um espaço temporal de 12 anos pode aumentar a fragilidade dos dados utilizados, sobretudo por mudanças no padrão de coleta, classificação dos dados e até mesmo da rotatividade de indivíduos que registram as informações 46. É importante destacar que as variáveis estado conjugal atual, consumo de álcool, tabagismo e origem do encaminhamento são de coleta opcional, o que implicou elevado percentual de dados faltantes 47 . Outro aspecto limitante é a ausência de variáveis clínicas que podem influenciar no desfecho estudado, dentre os quais estão os receptores hormonais e a imunohistoquímica, que são elementos indispensáveis para a instituição terapêutica. Já como fatores positivos deste estudo estão o tamanho da amostra analisada e a inclusão de unidades hospitalares de todas as regiões do Brasil, o que permitiu analisar o tempo para o acesso ao tra- 
tamento, especialmente no momento em que o Ministério da Saúde brasileiro instituiu portaria estabelecendo prazo para início do tratamento oncológico no país. As informações provenientes dos RHC são fundamentais para a identificação do perfil dos pacientes acometidos por neoplasias malignas e são uma importante ferramenta que possibilita a avaliação da qualidade da assistência oncológica no país.

Cabe ressaltar que, para maximizar a qualidade das informações dos RHC, são realizadas atualizações do sistema operacional, inclusive com o aprimoramento do conhecimento dos profissionais envolvidos na coleta, na digitação e no armazenamento dos dados.
Verificou-se que em $63,1 \%$ dos casos, o intervalo entre o diagnóstico e o tratamento foi de até 60 dias. Nas diversas regiões do Brasil, as mulheres mais suscetíveis ao atraso foram as idosas, não brancas, vivendo sem companheiro, com menos de oito anos de estudo, com doença em estadiamento inicial, que iniciaram o tratamento no período de 2006 a 2011 e oriundas do sistema público de saúde.

Faz-se necessária a realização de novas pesquisas direcionadas à identificação dos fatores associados aos longos intervalos de tempo nas etapas percorridas pelas mulheres com câncer de mama. Somente mediante identificação desses fatores será possível compreender as falhas existentes na abordagem dos casos de câncer de mama e desenvolver políticas de saúde pública específicas, centradas nas populações de maior risco. 


\section{Resumen}

Este estudio tuvo como objetivo analizar el intervalo de tiempo entre el diagnóstico y el inicio del tratamiento de cáncer de mama en las mujeres y sus determinantes. Este estudio de cohorte retrospectivo, involucró a 137.593 mujeres diagnosticadas, en 239 hospitales, en Brasil de 2000 y 2011. En el 63,1\% de los casos, el intervalo entre el diagnóstico y el tratamiento fue de 60 días. Las mujeres más propensas a retrasarlo eran: no blanca $(O R=$ 1,18; IC95\%: 1,13-1,23), sin pareja (OR = 1,05; IC95\%: 1,01-1,09), con menos de ocho años de educación (OR = 1,13; IC95\%: 1,08-1,18), con la enfermedad en una etapa temprana $(O R=1,27 ;$ IC95\%: 1,22-1,32), tratadas de 2006 a 2011 (OR = 1,54; IC95\%: 1,47-1,60) y dentro del sistema de salud pública (OR = 1,19; IC95\%: 1,13-1,25). Se observó entre las regiones de Brasil un análisis estratificado de los factores de variabilidad. La identificación de factores asociados con el retraso en la iniciación del tratamiento puede permitir la preparación de intervenciones propuestas a poblaciones específicas.

Neoplasias de la Mama; Tiempo de Tratamiento; Registros de Hospitales

\section{Colaboradores}

G. C. Medeiros contribuiu na concepção do projeto análise e interpretação dos dados e redação do artigo. A. Bergmann, S. S. Aguiar e L. C. S. Thuler participaram da concepção do projeto, análise e interpretação dos dados, revisão crítica relevante de conteúdo intelectual e aprovação da versão final a ser publicada.

\section{Referências}

1. Ferlay J, Soerjomataram I, Ervik M, Dikshit R, Eser S, Mathers C, et al. GLOBOCAN 2012 v. 1.0: cancer incidence and mortality worldwide. (IARC Cancer Base, 11). http://globocan.iarc.fr (acessado em 23/ Dez/2013).

2. Instituto Nacional de Câncer José Alencar Gomes da Silva. Estimativa 2014: incidência de câncer no Brasil. Rio de Janeiro: Instituto Nacional de Câncer José Alencar Gomes da Silva; 2013.

3. Instituto Nacional de Câncer José Alencar Gomes da Silva. Atlas de mortalidade por câncer. Rio de Janeiro: Instituto Nacional de Câncer José Alencar Gomes da Silva; 2011.

4. Instituto Nacional de Câncer. Controle do câncer de mama: documento de consenso. Rio de Janeiro: Instituto Nacional de Câncer; 2004.

5. Trufelli DC, Miranda VC, Santos MBB, Fraile NMP, Pecoroni PG, Gonzaga SFR, et al. Análise do atraso no diagnóstico e tratamento do câncer de mama em um hospital público. Rev Assoc Med Bras 2008; 54:72-6.
6. Coates AS. Breast cancer: delays, dilemmas, and delusions. Lancet 1999; 353:1112-3.

7. Sainsbury R, Johnston C, Haward B. Effect on survival of delays in referral of patients with breastcancer symptoms: a retrospective analysis. Lancet 1999; 353:1132-5.

8. Stiefel F. Understanding why women delay in seeking help for breast cancer symptoms. J Psychosom Res 2006; 60:309-10.

9. Ministério da Saúde. Portaria no 876, de 16 de maio de 2011. Dispõe sobre a aplicação da Lei no 12.732, de 22 de novembro de 2012, que versa a respeito do primeiro tratamento do paciente com neoplasia maligna comprovada no âmbito do Sistema Único de Saúde (SUS). Diário Oficial da União 2013; 17 mai.

10. Bleicher RJ, Ruth K, Sigurdson ER, Ross E, Wong YN, Patel SA, et al. Preoperative delays in the US medicare population with breast cancer. J Clin Oncol 2012; 30:4485-92. 
11. Oliveira EXG, Pinheiro RS, Melo ECP, Carvalho MS. Condicionantes socioeconômicos e geográficos do acesso à mamografia no Brasil, 2003-2008. Ciênc Saúde Coletiva 2011; 16:3649-64.

12. McLaughlin JM, Anderson RT, Ferketich AK, Seiber EE, Balkrishnan R, Paskett ED. Effect on survival of longer intervals between confirmed diagnosis and treatment initiation among low-income women with breast cancer. J Clin Oncol 2012; 30:4493-500.

13. Shin DW, Cho J, Kim SY, Guallar E, Hwang SS, Cho $\mathrm{B}$, et al. Delay to curative surgery greater than 12 weeks is associated with increased mortality in patients with colorectal and breast cancer but not lung or thyroid cancer. Ann Surg Oncol 2013; 20:2468-76.

14. Cunningham J, Hankey B, Lyles B, Percey C, Ries L, Seiffert J, et al. The SEER program code manual. Bethesda: National Cancer Institute; 1992.

15. Instituto Nacional de Câncer. TNM: classificação de tumores malignos. 6a Ed. Rio de Janeiro: Instituto Nacional de Câncer; 2004

16. World Health Organization. Active ageing: a police framework. Geneva: World Health Organization; 2002.

17. Williams DL, Tortu S, Thomson J. Factors associated with delays to diagnosis and treatment of breast cancer in women in a Louisiana urban safety net hospital. Women Health 2010; 50:705-18.

18. Caplan LS, May DS, Richardson LC. Time to diagnosis and treatment of breast cancer: results from the National Breast and Cervical Cancer Early Detection Program, 1991-1995. Am J Public Health 2000; 90:130-4.

19. Rezende MCR, Koch HA, Figueiredo JA, Thuler LCS. Causas do retardo na confirmação diagnóstica de lesões mamárias em mulheres atendidas em um centro de referência do Sistema Único de Saúde no Rio de Janeiro. Rev Bras Ginecol Obstet 2009; 31:75-81.

20. Trufelli DC, Bensi CG, Pane CEV, Ramos E, Otsuka FC, Tannous NG, et al. Onde está o atraso? Avaliação do tempo necessário para o diagnóstico e tratamento do câncer de mama nos serviços de oncologia da Faculdade de Medicina do ABC. Rev Bras Mastologia 2007; 17:14-8.

21. Mayo NE, Scott SC, Shen N, Hanley J, Goldberg MS, MacDonald NM. Waiting time for breast cancer surgery in Quebec. CMAJ 2001; 164:1133-8.

22. Maślach D, Krzyżak M, Szpak A, Owoc A, BielskaLasota M. Waiting time for treatment of women with breast cancer in Podlaskie Voivodeship (Poland) in view of place of residence. A population study. Ann Agric Environ Med 2013; 20:161-6.

23. Piñeros M, Sánchez R, Perry F, García AO, Ocampo $\mathrm{R}$, Cendales R. Demoras en el diagnóstico y tratamiento de mujeres con cáncer de mama en Bogotá, Colombia. Salud Pública Méx 2011; 53:478-85.

24. Vandergrift JL, Niland JC, Theriault RL, Edge SB, Wong YN, Loftus LS, et al. Time to adjuvant chemotherapy for breast cancer in National Comprehensive Cancer Network institutions. J Natl Cancer Inst 2013; 105:104-12.
25. Gagliato DM, Gonzalez-Angulo AM, Lei X, Theriault RL, Giordano SH, Valero V, et al. Clinical impact of delaying initiation of adjuvant chemotherapy in patients with breast cancer. J Clin Oncol 2014; 32:735-44.

26. Desch CE, McNiff KK, Schneider EC, Schrag D, McClure T, Lepisto E, et al. American Society of Clinical Oncology/National Comprehensive Cancer Network quality measures. J Clin Oncol 2008; 26:3631-7.

27. Gwyn K, Bondy ML, Cohen DS, Lund MJ, Liff JM, Flagg EW, et al. Racial differences in diagnosis, treatment, and clinical delays in a populationbased study of patients with newly diagnosed breast carcinoma. Cancer 2004; 100:1595-604.

28. Gorin SS, Heck JE, Cheng B, Smith SJ. Delays in breast cancer diagnosis and treatment by racial/ ethnic group. Arch Intern Med 2006; 166:2244-52.

29. Fedewa SA, Edge SB, Stewart AK, Halpern MT, Marlow N, Ward EM. Race and ethnicity are associated with delays in breast cancer treatment (2003-2006). J Health Care Poor Underserved 2011; 22:128-41.

30. Hansen RP, Olesen F, Sorensen HT, Sokolowski I, Sondergaard J. Socioeconomic patient characteristics predict delay in cancer diagnosis: a Danish cohort study. BMC Health Serv Res 2008; 8:49.

31. Baena-Cañada JM, Rosado-Varela P, EstalellaMendoza S, Expósito-Álvarez I, González-Guerrero M, Benítez-Rodríguez E. Influencia de los factores clínicos y biográficos en el retraso en iniciar la quimioterapia adyuvante en las pacientes con cáncer de mama. Med Clin (Barc) 2013; 140:444-8.

32. Seneviratne S, Campbell I, Scott N, Coles C, Lawrenson R. Treatment delay for Maori women with breast cancer in New Zealand. Ethn Health 2014; 20:178-93.

33. Lages RB, Oliveira GP, Simeão Filho VM, Nogueira FM, Teles JBM, Vieira SC. Desigualdades associadas à não realização de mamografia na zona urbana de Teresina-Piauí-Brasil, 2010-2011. Rev Bras Epidemiol 2012; 15:737-47.

34. Robertson R, Campbell NC, Smith S, Donnan PT, Sullivan F, Duffy R, et al. Factors influencing time from presentation to treatment of colorectal and breast cancer in urban and rural areas. Br J Cancer 2004; 90:1479-85.

35. Neal RD, Allgar VL. Sociodemographic factors and delays in the diagnosis of six cancers: analysis of data from the "National Survey of NHS Patients: Cancer". Br J Cancer 2005; 92:1971-5.

36. Leal MC, Gama SGN, Frias P, Szwarcwald CL. Healthy lifestyles and access to periodic health exams among Brazilian women. Cad Saúde Pública 2005; 21 Suppl 1:S78-88.

37. Matos JC, Pelloso SM, Carvalho MDB. Fatores associados à realização de prevenção secundária do câncer de mama no Município de Maringá, Paraná, Brasil. Cad Saúde Pública 2011; 27:888-98.

38. Ayala ALM. Sobrevida de mulheres com câncer de mama, de uma cidade do sul do país. Rev Bras Enferm 2012; 65:566-70. 
39. Eastman A, Tammaro Y, Moldrem A, Andrews V, Huth J, Euhus D, et al. Outcomes of delays in time to treatment in triple negative breast cancer. Ann Surg Oncol 2013; 20:1880-5.

40. Lima CA, Rangel MR, Macedo-Lima M, Silva AM. Time trends in breast cancer incidence and mortality in a mid-sized northeastern Brazilian city. BMC Public Health 2012; 12:883.

41. Oliveira EXG, Melo ECP, Pinheiro RS, Noronha CP, Carvalho MS. Acesso à assistência oncológica: mapeamento dos fluxos origem-destino das internações e dos atendimentos ambulatoriais. $\mathrm{O}$ caso do câncer de mama. Cad Saúde Pública 2011; 27: 317-26.

42. Silva GA, Bustamante-Teixeira MT, Aquino EML, Tomazelli JG, Santos-Silva I. Acesso à detecção precoce do câncer de mama no Sistema Único de Saúde: uma análise a partir dos dados do Sistema de Informações em Saúde. Cad Saúde Pública 2014; 30:1537-50.

43. Liedke PE, Finkelstein DM, Szymonifka J, Barrios $\mathrm{CH}$, Chavarri-Guerra Y, Bines J, et al. Outcomes of breast cancer in Brazil related to health care coverage: a retrospective cohort study. Cancer Epidemiol Biomarkers Prev 2014; 23:126-33.
44. Freitas-Júnior R, Gonzaga CMR, Freitas NMA, Martins E, Dardes RCM. Disparities in female breast cancer mortality rates in Brazil Between 1980 and 2009. Clinics 2012; 67:731-7.

45. Kluthcovsky ACGC, Faria TNP, Carneiro FH, Strona R. Female breast cancer mortality in Brazil and its regions. Rev Assoc Med Bras 2014; 60:387-93.

46. Pinto IV, Ramos DN, Costa MCE, Ferreira CBT, Rebelo MS. Completude e consistência dos dados dos registros hospitalares de câncer no Brasil. Cad Saúde Colet (Rio J.) 2012; 20:113-20.

47. Instituto Nacional de Câncer. Registros hospitalares de câncer: planejamento e gestão. 2a Ed. Rio de Janeiro: Instituto Nacional de Câncer; 2010.

Recebido em 26/Mar/2014

Versão final reapresentada em 14/Nov/2014

Aprovado em 09/Jan/2015 